\title{
Relación de las variables organizacionales y la gestión del talento humano, en las hosterías de ciudad riobamba, Ecuador
}

\section{RESUMEN}

El presente artículo refleja resultados de la investigación, al determinar el grado de influencia de la gestión del talento humano en hosterías sobre el desarrollo sostenible del turismo en el cantón Riobamba, Ecuador. El tipo de investigación es de enfoque cuantitativo, aplicada, descriptiva, correlacional no experimental y transversal. Los objetivos logrados son de relacionar proceso de gestión en el campo de acción de las hosterías, Demostrar la necesidad de gestionar desde la visión de la sostenibilidad inscrito en la competitividad empresarial. Se realizo 44 cuestionarios para trabajadores y empleados de las hosterías conformados por 32 ítems, los resultados obtenidos con el Alfa de Cronbach cuyo valor fue 0.872 , este se evalúa como bueno por tanto el instrumento es fiable para ser aplicado, por lo que se concluye que existe correlación significativa de acuerdo a la muestra; la mayor correlación viene dada entre las dimensiones de cultura organizacional y diseño organizacional con un porcentaje de 0.979 , y la peor correlación está dada por el conocimiento y valores con un porcentaje de 0.102

Palabras Claves: Sostenibilidad del Turismo; Gestión del Talento Humano; Cultura Organizacional; Diseño Organizacional; Conocimiento; Valores

RELATIONSHIP AMONG THE ORGANIZATIONAL VARIABLES AND THE HUMAN TALENT MANAGEMENT IN RIOBAMBA'S HOSTELRIES, ECUADOR

\section{ABSTRACT}

This article presents the results of the research in determining the influence degree in human management in Hostelries about sustainable tourism in Riobamba city, Ecuador. The research has quantity focus, descriptive, correlational; it is not experimental and transversal. The objectives achieved are: To related the field management processes in action in the hostelries. To show the needs that management from the sustainability view in the business competitively. It was developed 44 questionnaires to be applied with workers, employers from the hostelries. The results getting from the research through the Alpha Cronbach its value was 0.872 it is considered as good; therefore the instrument is reliable to be applied. In conclusion, there is a significant correlation according to the sample; the biggest correlation comes between the dimension of organizational culture and the organizational design with a percentage of 0.979 and the worst comes from by the knowledge and its value with a percentage of 0.102

Keywords: Tourism Sustainability; Management of Human Talent; Organizational Culture; Organizational Design; Knowledge; Values.

\section{INTRODUCCION}

A tenor de Aldaz, (2017, p.12), "relacionar términos tales como "competitividad" y "sostenibilidad" en las organizaciones, -fundamentalmente de aquellas orientas a la gestión del turismo y hotelería-, implica adoptar la gestión del talento humano como proceso clave del éxito organizacional." La gestión del talento humano, -asumiendo los postulados de Alles, (2008), resulta herramienta implícita en el proceso de toma de las decisiones y reorientaciones estratégicas para las empresas, especialmente para los establecimientos de alojamiento tomando en cuenta que el personal que labora en estos lugares, -es decir el talento humano contratado-, brindan una atención personalizada desde el nivel técnico, encargándose de la ejecución de las tareas, hasta la junta de accionistas que tienen a su cargo el control total. Existen cuatro elementos importantes que una organización debe tener en consideración, los recursos materiales, financieros, humanos, tecnológicos y la sostenibilidad.

Los recursos materiales son los bienes tangibles con los que cuenta la empresa para ofrecer un mejor servicio a sus clientes y en el menor tiempo posible, los recursos financieros son propios o ajenos y son parte fundamental para el buen funcionamiento y desarrollo de la empresa, los recursos humanos son el elemento esencial sin ellos no existirá una adecuada administración, - asumiendo los enfoques de Becker y Gerhart, (1996)-, los recursos tecnológicos, hace posible la información y comunicación para satisfacer las necesidades del cliente, y la sostenibilidad que asegura las necesidades del presente sin comprometer las necesidades de futuras generaciones. OMT (1994).

Las Hosterías del cantón Riobamba denotan una deficiente gestión del talento humano con prácticas y políticas inadecuadas para vincular el conocimiento, habilidad, Actitud, Valores, Cultura organizacional, diseño organizacional y estilo de organización, además se debe considerar las relaciones humanas, liderazgo, trabajo en equipo y el desarrollo del turismo sostenible, -todo ello apelando a los enfoques de Beer (1997) que permita identificar necesidades, expectativas, exigencias y competitividad, en primera instancia otorgará un alto nivel de desarrollo laboral,

1 Institución: Universidad Nacional del Chimborazo. Docente Investigador e-mail: saldaz@unach.edu.ec

2 Institución: Universidad Nacional del Chimborazo. Docente Investigador e-mail: hpacheco@unach.edu.ec

3 Institución: Universidad Nacional del Chimborazo. Docente Investigador e-mail: vvelasco@unach.edu.ec

4 Institución: Universidad Nacional del Chimborazo. Docente Investigador e-mail: dcalvopina@unach.edu.ec 
frente al desarrollo empresarial, para así garantizar un ambiente seguro y equitativo en las hostelerías de Riobamba . Por ello, de los objetivos propuestos en la investigación, está el de relacionar procesos de gestión como es el talento humano en el campo de acción de las hosterías, y la influencia de este proceso de talento humano en la sostenibilidad turística, tomando como caso de estudio la provincia de Chimborazo.

Además de alcanzar este citado objetivo, se citan como objetivos colaterales el evidenciar y asumir el enfoque de transdisciplinariedad al asumir la sostenibilidad del turismo y el proceso de gestión del talento humano, ello tomar del enfoque de gestión y los principios de la sostenibilidad turística sus postulados e imbricar estos en una sola función. Por otro lado, se exige demostrar la necesidad de gestionar el talento humano desde la visión de la sostenibilidad inscrito en la competitividad empresarial, puesto que ya los términos puramente económicos y organizacionales exigen de la identificación con el enfoque de la sostenibilidad, -en este caso turística-.

De la importancia y necesidad de este trabajo investigativo está el proveer herramientas y conocimientos que permitan al gestor hotelero perfeccionar su trabajo, además de incorporar el estado del arte en materia gerencial a su labor, asumiendo además los criterios de Castro (2012). Nada tan alejado de la realidad, -a criterio de esta autora-, que el enfoque tecnológico pretendiendo alcanzar estándares de clase mundial, obviando el prisma humano, visibilizado en la gestión del talento humano. Del campo de acción seleccionado, -la ciudad de Riobamba, perteneciente a la provincia Chimborazo-, resulta un clásico ejemplo por los empresarios de la adquisición de tecnologías e inversión hotelera, más no existen antecedentes de gestión del talento humano, asumiendo estos empresarios que solo basta estar graduado de instituto o universidad con carreras de turismo y hotelería, obviando gestionar estos especialistas y personal en general.

\section{METODOLOGÍA}

Como investigación declarada de corte científico, son adoptadas las investigaciones del tipo cuasi-experimental de tipo transversal y relacional causal. Ello se fundamenta es que con la primera obtenemos una visión general y luego proseguir con un estudio de caso, para así centrarse en las razones subyacentes de los resultados generado. Respecto a la decisión de lo transversal, es que se recolectan datos en un solo momento, se describen variables y se realiza su relación en ese momento como lo menciona: Lai, Liu, Tucker, Sane y Greenberg, (2008). Su propósito es describir varia- bles y analizar su incidencia e interrelación en un momento dado, es como tomar una fotografía de algo que sucede. ya lo relacional causal se revela ya que su propósito es describir las relaciones entre dos o más categorías, conceptos o variables en un momento determinado, se trata de describir relaciones, pero además precisar sentido de causalidad. La investigación es un tipo de estudio que tiene como propósito evaluar la relación que existe entre dos o más conceptos, categorías o variables (en un contexto en particular). A tenor de Hernández, Fernández y Baptista, (2014). "Los estudios cuantitativos correlacionales, miden el grado de relación entre dos o más variables (cuantifican relaciones), miden cada variable presuntamente relacionada y después también miden y analizan la correlación. Tales correlaciones se expresan en hipótesis sometida a prueba". (p.121).

Así, el diseño de la Investigación de acuerdo al tipo de investigación adoptado (descriptivo explicativo) el presente estudio aplica un diseño "descriptivo correlacional". De las técnicas empleadas, se cita la Observación Para el estudio del problema planteado en las hosterías en primer lugar se analizó mediante observación, esta técnica consiste en verificar el comportamiento en los puestos de trabajo de los empleados y trabajadores de los establecimientos de alojamiento. Asimismo, la encuesta a la hora de recopilar los datos en la presente investigación para las dos muestras. Otra herramienta resulta el cuestionario a la hora de evaluar la Gestión del Talento Humano, -apelando a los estudios de Chiavenato (2009)-, considerando las dimensiones tales como Conocimientos; Habilidades; Actitudes; Valores; Cultura Organización; Diseño Organizacional; Estilo de Administración

Para procesar la información se clasificaron, registraron y codificaron los datos para luego ser analizados con el Software estadístico informático (IBM SPSS Versión 23: Statistics) y su extensión AMOS. Al medir el constructor de gestión del talento humano se utilizó un cuestionario ya validado. Se trata de un instrumento diseñado con la técnica de Likert, comprendido en su versión final de un total de 32 ítems vinculados a las 7 dimensiones explicadas anteriormente. Al pretender medir los Modelos de Ecuaciones Estructurales, se apela al análisis multivariante y son particularmente de gran ayuda en las Ciencias Sociales y del comportamiento. Estos suelen usarse en el estudio de las relaciones entre las áreas sociales y los logros obtenidos; por ejemplo, la discriminación en los empleos, la eficacia de programas de acción social, etc.

Al analizar algunos indicadores de ajustes de un buen modelo, y considerando las investigaciones Cruz (2013), el que arroja mejores resultados es el método de MV. Referido a los métodos científicos 
asumidos en esta investigación, se citan el método de expertos, acudiendo a empresarios y académicos de amplia experiencia para evacuar criterios y dudas en la ejecución del trabajo de campo, método de las contradicciones dialécticas, revelando la génesis y causas que generan la idea del trabajo, -señalado esto por Cuesta (2002)-, señalando entre otras contradicciones la disposición del estado ecuatoriano en potenciar el turismo y la insuficiente investigación e interrelación entre procesos de gestión para la sostenibilidad del turismo, de las inversiones en tecnologías y estado del arte en materia de confort turístico y la ausencia del enfoque de gestión del talento humano orientado a la sostenibilidad del turismo, método de análisis y síntesis de la bibliografía estudiada, métodos empíricos y heurísticos al aplicar los instrumentos de gestión informacional, métodos estadísticos matemáticos al procesar la información acopiada.

\section{RESULTADOS Y DISCUSIÓN}

Inicialmente, se realiza el análisis e interpretación de los resultados obtenidos, - y a tenor de George y Mallery (2003), expresados en tablas de Máxima Verosimilitud (MV), Mínimos Cuadrados Generalizados (MCG) y Mínimos Cuadrados de Libre Escala (MCLE) y gráficos. Es ejecutado el análisis de las 7 dimensiones de la variable de la gestión del talento humano las mismas que son: conocimientos, habilidades, actitudes, Valores. Cultura organizacional, -apelando a Schein (1988)-, diseño organizacional y estilo de administración, para ello se ejecutó el análisis de fiabilidad y consistencia interna de la escala de aptitud de tipo Likert aplicada al cuestionario, lo hacemos a través del estadístico Alfa de Cronbach, la cual permite estimar la fiabilidad de un instrumento de medida a través de un conjunto de ítems que se espera que midan el mismo constructo o dimensión teórica.

La medida de la fiabilidad mediante el alfa de Cronbach asume que los ítems (medidos en escala tipo, Likert) miden un mismo constructo y que están altamente correlacionados. Cuanto más cerca se encuentre el valor del alfa a 1 mayor es la consistencia interna de los ítems analizados. La fiabilidad de la escala debe obtenerse siempre con los datos de cada muestra para garantizar la medida fiable del constructo en la muestra concreta de investigación.

Como criterio general, a tenor de (George y Mallery, 2003, p.231), se adoptan sus recomendaciones para evaluar los coeficientes de alfa de Cronbach. El coeficiente alfa de Cronbach al ser 0.872 , se evalúa como bueno, con 32 elementos, por tanto, el instrumento es fiable para ser aplicado.
Para realizar la validación del constructo, se aplicó el AFC conocido como Análisis Factorial Confirmatorio. El análisis factorial consta de cuatro fases características: el cálculo de una matriz capaz de expresar la variabilidad conjunta de todas las variables, la extracción del número óptimo de factores, la rotación de la solución para facilitar su interpretación y la estimación de las puntuaciones de los sujetos en las nuevas dimensiones.

Estudiando las comunalidades de la extracción podemos valorar cuáles de las variables son peor explicadas por el modelo. En nuestro ejemplo la mayor correlación viene dada entre las dimensiones de cultura organizacional y diseño organizacional con un porcentaje de 0.979 , y la peor correlación está dada por el conocimiento y valores con un porcentaje de 0.102 . Al realizar dicho análisis por el Método de extracción de Máxima Verosimilitud y Rotación Oblimin Directo, el AFC muestra inconsistencias debido a que no calcula la Matriz de $\mathrm{Pa}-$ trón y Matriz de Estructura, cosa que imposibilita obtener los resultados de las dimensiones de igual forma, el valor de KMO está por debajo de 0.5 algo que pone en duda el procedimiento para este caso.

En nuestra investigación, el valor que asume el $\mathrm{KMO}=0.426$, lo que pone en incertidumbre en este caso por lo que el Análisis Factorial aplicado a las variables no es efectivo debido a que el tamaño muestral es pequeño. El valor de significación de la prueba de esfericidad de Bartlett toma un valor 0.000 , mucho menor que el nivel teórico de significación alfa de 0.05 , de esta forma rechazamos la hipótesis nula de esfericidad, por lo que el modelo factorial es adecuado.

Abordando los Modelos de Ecuaciones Estructurales, se corren dos métodos de estimación sobre el modelo teórico, resueltos por el método de Máxima Verosimilitud (MV de ahora en adelante) y Mínimo Cuadrados Generalizados (MCG de ahora en adelante):

\section{Indicadores sobre bondad del ajuste gestión del talento humano}

MV - CMIN: $896.95 ;$ NFI: 0.323 ; PNFI: 0.289;
GFI: 0.540; PGFI: 0.453

MCG - CMIN: 375.17; NFI: 0.198; PNFI: 0.177; GFI: 0.455; PGFI: 0.381

Por lo que se ha utilizado para el modelo teórico 2 tipos de métodos de solución, no fue posible analizar otros debido al tamaño muestral. Al analizar algunos indicadores de ajustes de un buen modelo, el que arroja mejores resultados es el método de MV. El análisis se hará sobre este modelo. Ver figura 1. 


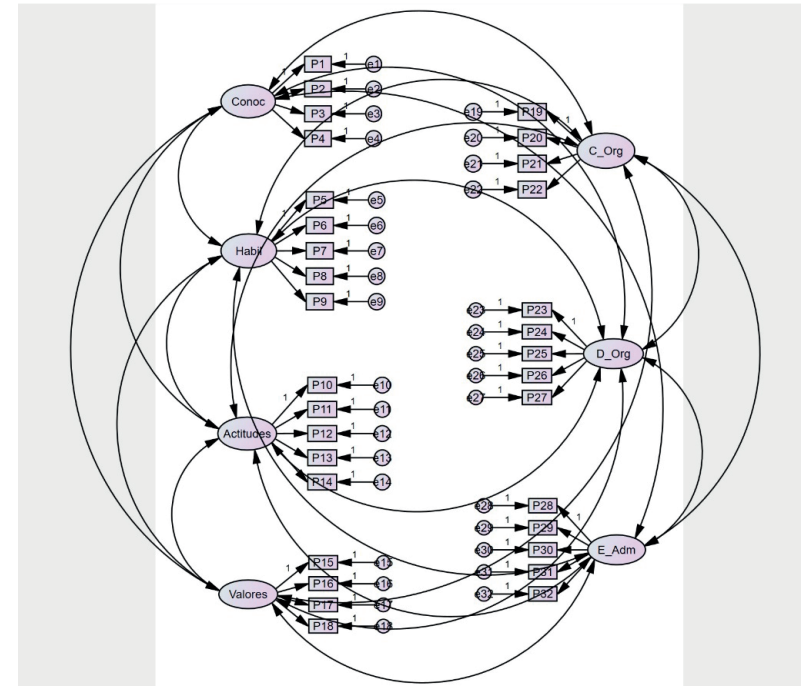

Figura 1. Modelo teórico gestión del talento humano Fuente: Elaboración propia

Se parte de analizar las estimaciones infractoras, hay una varianza correspondiente a e1 que es negativa, por lo que es probable que se eliminen variables del modelo.

Luego de un profundo análisis de los resultados, resulta clara la eliminación de las variables $\mathrm{P}_{1}, \mathrm{P}_{4}$, $P_{9}, P_{11}$ y $P_{12}$, de esta forma se logra estabilizar tanto las estimaciones estandarizadas como las varianzas de los errores negativas, y por ende, los estadísticos de Bondad de Ajuste por lo que en el figura 2 que se propone a continuación se eliminan esas variables y se expresa de la siguiente manera:

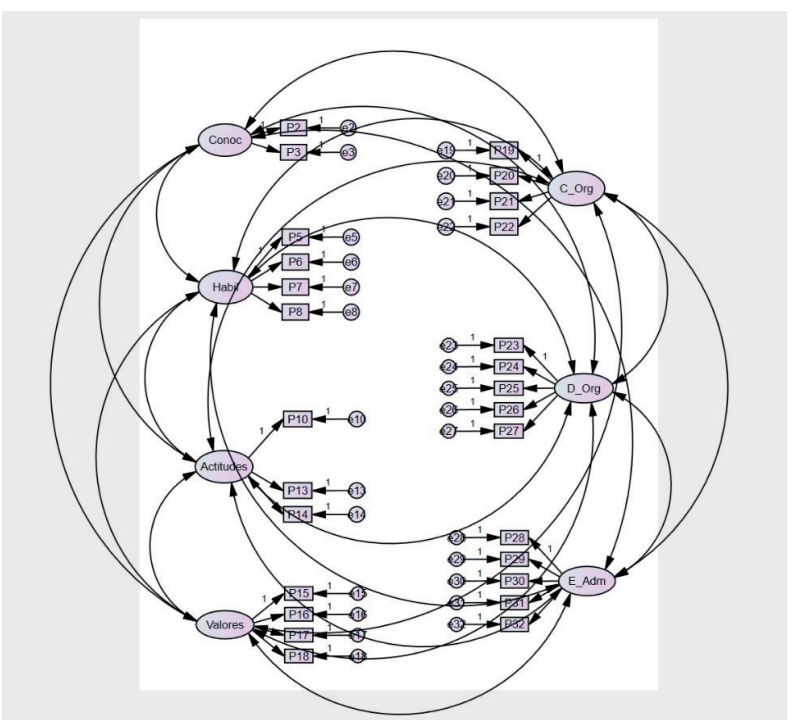

Figura 2. Modelo Estructural Mejorado gestión del talento humano

Fuente. Elaboración propia

Evaluando los indicadores de bondad de ajuste modelo teórico mejorado gestión del talento humano, se aprecia que, estos son mejores a los del modelo teórico, pero aún no satisfacen los criterios de buen modelo, pero esto está influenciado con el tamaño de la muestra. Se buscará partiendo de este modelo mejorado calculado, un modelo que permita mejorar aún más los resultados obtenidos, el único posible es el método de Mínimos Cuadrados Libre de Escala. (MCLE de ahora en adelante). Se procedió al cálculo del modelo, y los resultados fueron más alentadores, esto se muestra a continuación.

Indicadores de bondad de ajuste modelo teórico mejorado gestión del talento humano

MV - CMIN: 896.95; NFI: 0.323; PNFI: 0.289; GFI: 0.540; PGFI: 0.453

CMIN: 552.02; NFI: 0.374; PNFI: 0.323; GFI: 0.609; PGFI: 0.488

Indicadores de Bondad de ajuste mínimos cuadros libre de escala gestión del talento humano mejorado.

MV- CMIN: 896.95; NFI: 0.323; PNFI: 0.289; GFI: 0.540; PGFI: 0.453

MCL- CMIN: 246.36; NFI: 0.763; PNFI: 0.658; GFI: 0.848; PGFI: 0.680

Se puede apreciar como mejoran los indicadores de Bondad de Ajuste, y como disminuye el valor de Chi-Cuadrado, por lo que el análisis se hará respecto al modelo Mejorado por el Método de Mínimos Cuadrados Libres de Escala.

Medidas de Ajuste Absoluto. Evaluación del Modelo Global, índice de la Chi-Cuadrado: Analiza la hipótesis nula de que el modelo es no significativo. Válido para muestras pequeñas.

Índice de bondad del ajuste (GFI): Su valor está comprendido entre 0 y 1 , indicando este último un ajuste perfecto.

Índice de Raíz Cuadrada Media del Error de la Aproximación (RMSEA): La evaluación de su magnitud es subjetiva considerándose que un menor valor que 0.05 es un indicativo de buen ajuste, aunque autores plantean que bastaría con que esté por debajo de 0.084 .

Medidas de Ajuste Incremental. Evaluación del Modelo Global.

Índice de Ajuste Normalizado (NFI): Mide la reducción proporcional en la función de ajuste cuando pasamos del modelo nulo al propuesto. Los valores de este índice varían entre 0 y 1 , considerándose aceptables valores mayores a 0.9 .

Índice de Ajuste no Normalizado (NNFI o TLI): Es un índice que supera las limitaciones del Índice de Ajuste Normalizado al considerar los grados de libertad del modelo propuesto y nulo, estando por tanto muy débilmente relacionado con el tamaño 
muestral. El rango de este índice varía entre 0 y 1 , siendo recomendables valores mayores a 0.9 .

Índice de Ajuste Comparativo (CFI): Mide la mejora en la medición de la no centralidad de un modelo. La medida oscila entre 0 para un modelo mal ajustado y 1 para un modelo bien ajustado.

Medidas de Ajuste de Parsimonia. Evaluación del Modelo Global.

Criterio de Información de Akaike (AIC): Es un índice comparativo entre modelos, cuyos valores próximos a 0 indican un buen ajuste.

Índice de Ajuste Parsimónico Normalizado (PNFI): Constituye una modificación del índice de ajuste normalizado incorporada por la razón entre los grados de libertad de los dos modelos alternativos que se pretenden comparar. Los valores elevados del PNFI son mejores. Diferencias mínimas de 0.06 a 0.09 serían necesarias para indicar cambios sustanciales en los modelos.

Índice de Bondad del Ajuste Parsimónico (PGFI): Presenta una modificación del Índice de Bondad del Ajuste (GFI). Los valores se establecen de 0 a 1 , siendo los valores más próximos a 1 los que indiquen mayor equilibrio (parsimonia) en el modelo.

\section{Evaluación del Modelo de Medida.}

- Validez Convergente de cada uno de los factores latentes: Cargas factoriales mayores que 0.6 .

- Uni-dimensionalidad de cada factor latente:

Los indicadores tienen un ajuste aceptable sobre un modelo de un único factor: $t$ asociado con cada una de las ponderaciones, así como los índices de ajustes del modelo presentan valores adecuados. Correlaciones entre los distintos factores latentes: si se observa que la relación entre algunos de los factores fuera alta (superior a 0.5), podríamos cuestionarnos la validez discriminante de sus escalas de medida. Análisis de fiabilidad de los distintos constructos con el fin de evaluar si los indicadores especificados para los mismos son suficientes en su representación.

Referido a la evaluación del Modelo Estructural, se identifican si todos los estimadores del modelo son significativos; si alguno no lo fuese, se debería suprimir la relación entre la variable latente teniendo en cuenta la teoría. Se aprecia en los resultados que los modelos estimados por MV y MCLE, presentan resultados coherentes por lo que la decisión de cuál modelo aceptar vendrá especificado por los análisis de las alfas de Cronbach tanto para las preguntas que componen las dimensiones teóricas, como para las que planea el AFC, tal y como se refleja en la Tabla 1.

Se comenzará por analizar los Alfas de Cronbach.

Tabla 1. Análisis alfas de las dimensiones del modelo teórico y del modelo mejorado

\begin{tabular}{lcccc}
\hline & \multicolumn{2}{c}{ Modelo teórico } & \multicolumn{2}{c}{ Modelo mejorado } \\
\hline $\begin{array}{c}\text { Dimensiones } \\
\text { de Gestión } \\
\text { del Talento } \\
\text { Humano }\end{array}$ & $\begin{array}{c}\text { Alfa de } \\
\text { Cronbach }\end{array}$ & $\begin{array}{c}\mathrm{N}^{\circ} \text { de } \\
\text { elementos }\end{array}$ & $\begin{array}{c}\text { Alfa de } \\
\text { Cronbach }\end{array}$ & $\begin{array}{c}\mathrm{N}^{\circ} \text { de } \\
\text { elementos }\end{array}$ \\
\hline Conocimiento & 0.682 & 4 & 0.743 & 2 \\
\hline Habilidades & 0.587 & 5 & 0.647 & 4 \\
\hline Actitudes & 0.359 & 5 & -0.062 & 3 \\
\hline Valores & 0.369 & 4 & 0.369 & 4 \\
\hline $\begin{array}{l}\text { Cultura Orga- } \\
\text { nizacional }\end{array}$ & 0.672 & 4 & 0.672 & 4 \\
\hline $\begin{array}{l}\text { Diseño Orga- } \\
\text { nizacional }\end{array}$ & 0.640 & 5 & 0.640 & 5 \\
\hline $\begin{array}{l}\text { Estilo organi- } \\
\text { zacional }\end{array}$ & 0.798 & 5 & 0.798 & 5 \\
\hline
\end{tabular}

Fuente. Elaboración propia

En el modelo mejorado el alfa de Cronbach de la dimensión de Actitudes es negativo, debido a una covarianza promedio negativa entre elementos. Esto viola los supuestos del modelo de fiabilidad. A través de estos resultados se concluye que es mejor trabajar con el modelo teórico que con el modelo mejorado ya que el valor del Modelo de ecuaciones estructurales (SEM), el alfa es negativo en el modelo mejorado.

Todo lo expresado demuestra fehacientemente que existe la relación entre la gestión del talento humano y las variables seleccionadas, además de ilustrar el grado de relación de cada una, ello es, la validación del presupuesto teórico.

\section{CONCLUSIONES}

Asumiendo los objetivos previamente declarados, y que corresponden a la investigación, se concluye que:

- Han sido relacionados y validado el grado de relación de los procesos de gestión del talento humano en el campo de acción de las hosterías, y la influencia de este proceso en la sostenibilidad turística, tomando como caso de estudio la provincia de Chimborazo.

- Se evidencia al asumir el enfoque de transdisciplinariedad al converger los postulados de la sostenibilidad del turismo y las practicas inherentes al proceso de gestión del talento humano, imbricados ambos en una sola función. 
- Logra demostrarse la necesidad de gestionar el talento humano desde la visión de la sostenibilidad inscrito en la competitividad empresarial, exigiendo que estos términos puramente económicos y organizacionales gocen de la identificación con el enfoque de la sostenibilidad turística.

\section{SE RECOMIENDA:}

- Adoptar estos resultados científicos investigativos por la dirección académica de la UNACH, integrando los saberes a la malla curricular de la carrera de licenciatura gestión turística y hotelera.

- Propiciar talleres y asesorías a entidades públicas y privadas, enfatizando en la gestión del talento humano orientado a la sostenibilidad del turismo.

- Gestar temas de investigación en los Proyectos Integradores de Saberes, (PIS) de los diferentes semestres de la carrera de Gestión Hotelera y Turística, donde sostenibilidad del turismo y gestión del talento humano se visibilice

\section{AGRADECIMIENTO}

Universidad Nacional del Chimborazo.

- Universidad de San Marcos, facultad de Ingeniería Industrial

- Hosterías Ciudad de Riobamba

- Doctora Durga Edelmira Ramírez

\section{REFERENCIAS BIBLIOGRÁFICAS}

[1] Aldaz Hernández Silvia Marieta. (2017): La Gestión del talento Humano en las Hosterías de Riobamba orientadas al Desarrollo Sostenible del Turismo en el Ecuador. Universidad nacional Mayor de San Marcos. Facultad Ingeniería Industrial. Lima, Perú.

[2] Alles, M. A. (2009): Diccionario de competencias (Vol. 1). Buenos Aires, Argentina: Ediciones Granica S.A.

[3] Becker, B. \& Gerhart, B. (1996): The impact of human resource management on organizational performance: Progress and prospects. Academy of Management Journal, 39(4), 779801.
[4] Beer, M. (1997): The transformation of the human resource function: Resolving the tension between a traditional administrative and a new strategic role. Human Resource Management, 36(1),49-56.

[5] Castro M. L. A. (2012): Formación y desarrollo de competencias gerenciales para los gestores hoteleros. Cuadernos de Administración 28(48), 64-76

[6] Chiavenato, I. (2009): Comportamiento organizacional. La dinámica del éxito de las organizaciones. (2 ${ }^{\mathrm{a}}$. Ed.). Editorial Mc Graw Hill. México.

[7] Cruz L. M. E. (2013): Gestión de los recursos humanos por competencias en el desarrollo del sector turístico. El caso de Ambato: Amenazas y oportunidades. Tesis de grado doctoral. Universidad Rey Juan Carlos. España.

[8] Cuesta S (2002): Gestión del Conocimiento, Análisis y Proyección de los Recursos Humanos. La Habana, Cuba: Editorial Academia, Habana.

[9] George, D., \& Mallery, P. (2003): Reliability analysis. SPSS for Windows, step by step: a simple guide and reference, 14th edn. Boston: Allyn \& Bacon, 222-232.

[10] Hernández, R., Fernández C. y Baptista P. (2014): Metodología de la Investigación. (6ta ed.). México: Editorial Mc Graw Hill, interamericana editors, S.A

[11] Lai, T. S., Liu, Y., Tucker, T., Daniel, K. R., Sane, D. C., Toone, E., \& Greenberg, C. S. (2008): Identification of chemical inhibitors to human tissue transglutaminase by screening existing drug libraries. Chemistry \& biology, 15 (9), 969-978.

[12] OMT. Bordas, E. (1994) "La calidad de los servicios turísticos de la teoría a la práctica”. En: Seminario de la organización mundial de turismo sobre la calidad, un reto para el turismo. Madrid. Organización Mundial de Turismo

[13] Schein, E. (1988): La cultura empresarial y el liderazgo. Una visión dinámica. (1. Ed.) P. imprenta. Barcelona, España Plaza \& Janés. 